\title{
sciendo
}

DOI: 10.1515/aon-2019-0001

\section{ACQUiSITION AND TRACKING PERFORMANCE OF SATELlite NAVIGATION SYSTEM SIGNAL USING TIERED DIFFERENTIAL POLYPHASE CODE}

\author{
GwangHee Jo \\ dept. electronics engineering \\ Chungnam National University \\ Daejeon, Republic of Korea \\ j_ghee@cnu.ac.kr \\ JuHyun Lee \\ dept. Radio Navigation \\ Navcours \\ Daejeon, Republic of Korea \\ juhyun8878@navcourse.채— \\ JaeHee Noh \\ dept. electronics engineering \\ Chungnam National University \\ Daejeon, Republic of Korea \\ jhnoh3555@cnu.ac.kr \\ SangJeong Lee \\ dept. electronics engineering \\ Chungnam National University \\ Daejeon, Republic of Korea \\ eesj1@cnu.ac.kr \\ JinHyuk Lee \\ dept. electronics engineering \\ Chungnam National University \\ Daejeon, Republic of Korea \\ jh_lee@cnu.ac.kr
}

\begin{abstract}
In this paper, we analyze the acquisition and tracking performance of signal using a tiered differential polyphase code as the secondary code. The Zadoff-Chu sequence is known to have a CAZAC (Constant Amplitude Zero Auto-Correlation) characteristics. The secondary code generated by differential encoding of the Zadoff-Chu sequence also has the same characteristics as the Zadoff-Chu sequence. Therefore, long integration will give better correlation results. We compare signal acquisition and tracking performance when using the NH sequence and Zadoff-Chu sequence as the secondary code. Monte-carlo simulation is performed using MATLAB. We use the probability of detection and the mean acquisition time for signal acquisition performance and tracking jitter for signal tracking performance.
\end{abstract}

Keywords: Zadoff-Chu sequence, polyphase code, acquisition, tracking 


\section{INDRUCTION}

The GNSS signal arriving at the receiver may become weaker if interfered with by other signals or blocked by obstacles such as buildings or mountains[1]. Modernized GNSS signals have different characteristics from conventional GPS L1 C / A signals to improve SNR. Modernized GNSS signals may use longer codes or separate pilot and date channels to increase integration time[2]. In the first method, the longer code is generated by increasing the length of the PRN code and by using the secondary code together with the PRN code (primary code). In the second method, the pilot channel does not transmit data, so no bit transition occurs. Therefore, the integration time can be increased to improve the SNR. It also has the advantage of using pure PLLs. GNSS can combine these two methods.

Generally, secondary codes used in GNSS are generated by combining 1 and 0 . However, a secondary code using the Zadoff-Chu sequence, a polyphase code, has also been proposed[3]. This secondary code is Tiered Differential Polyphase Code(TDPC). As a result, the phase of the $1 \mathrm{~ms}$ correlation value changes. The receiver is characterized by multiplying the secondary code conjugate to wipe off the secondary code. Currently there is no GNSS that uses this polyphase code as a secondary code.

In this paper, we adopt the TDPC and the Neumann-Hoffman (NH) code of the same length as the secondary code. To compare only the effect of the secondary code, the simulation is performed assuming that the primary code is the GPS L1 C / A code. We compare signal acquisition performance over integration time. The signal tracking is performed at $1 \mathrm{~ms}$ intervals and compares the performance according to the secondary code. We use the probability of detection for signal acquisition performance and tracking jitter for signal tracking performance. Using these results, we compare the effect of secondary code on signal acquisition and tracking.

\section{TIERED DIFFERENTIAL POLYPHASE CODE}

The Zadoff-Chu sequence of length $N$ consists of complex numbers as following;

$$
a_{k}=\operatorname{expi\alpha } \alpha_{k} \quad k=0,1, \cdots, N-1 .
$$

Considering the case where $N$ is even, equation (1) can be rewritten as follows[4]

$$
a_{k}=\exp i \frac{M \pi k^{2}}{N}
$$

where $M$ is an integer and $M$ and $N$ are coprime. If the phases of the sequences are different, the autocorrelation result is

$$
x_{j}=\sum_{k=0}^{N-j-1} a_{k} a_{k+j}^{*}+\sum_{k=N-j}^{N-1} a_{k} a_{k+j-N}^{*}=0, j=1,2, \cdots N-1 .
$$

The secondary code generated by differential encoding of the Zadoff-Chu sequence is given as[3]

$$
C_{s}(k)=\left\{\begin{array}{cc}
a_{k} & k=0 \\
a_{k} C_{s}(k-1) & 0<k \leq N-1
\end{array} .\right.
$$


The differential decoding process is performed with the correlation value after despreading the primary code. When $u(n)$ is defined as the correlation value calculated for $1 \mathrm{~ms}, u(n)$ is given as

$$
u(n)=A d C_{s}(n)
$$

where only the Zadoff-Chu sequence is left in the equation. Therefore, the phase of the secondary code can be found by accumulating the conjugate of the Zadoff-Chu sequence multiplied by the above equation for $20 \mathrm{~ms}$. Then the navigation message can be demodulated by despreading the secondary code.

The proposed TDPC length is 20 . The NH sequence $(0,0,0,0,0,1,0,0,1,1,0,1,0,1,0,0,1,1,1,0)$ is used for Beidou and has a length of 20. TDPC and NH sequence have the same length and are generated at 1 kbps[5].

\section{SIMULATION}

This section summarizes the simulation results. In simulation using MATLAB, we assume that the receiver receives 7 visible satellites signals. The signal strength was set at $-169 \mathrm{dBW}$, about $10 \mathrm{~dB}$ lower than the GPS nominal power. As mentioned earlier, we have created a GPS L1 C / A code with and without secondary code. The secondary codes are the NH sequence and the TDPC. It is assumed that the Doppler frequency is known. The signal acquisition threshold is determined so that the probability of false alarm is $0.1 \%$. we compared the probability of detection and the mean acquisition time according to the secondary code. Signal tracking is performed at intervals of $1 \mathrm{~ms}$ after removing the secondary code. The simulation platform is shown in Fig. 1.

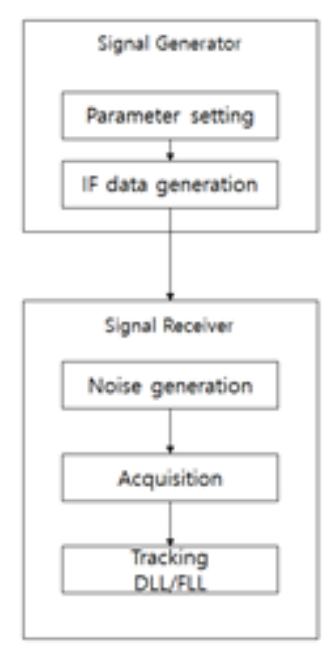

A. Signal Acquisition

Fig. 1. Simulation platform.

Since the signal strength is set to $-169 \mathrm{dBW}$, this is a weak signal environment. Signal acquisition performance with integration time was analyzed. The integration time is 1, 2, 4, 5, 10, $20 \mathrm{~ms}$ considering the data bit duration. The data bits are assumed to be aligned. Therefore, bit transitions do not occur. In this condition we analyze the signal acquisition performance. The FFT / IFFT was used to calculate the correlation value to determine whether the signal was acquired and is shown in Fig. 2. 


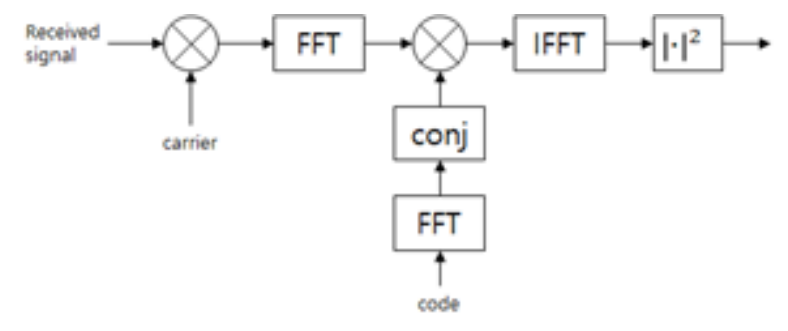

Fig. 2. FFT Correlation.

The probability of false alarm is set to $0.1 \%$ when there is noise only. The equation is as following

$$
P_{f a}=\int_{V_{t}}^{\infty} p_{n}(z) d z
$$

In Eq. (7), $p_{n}(z)$ is the pdf of the envelope with the signal absent. The signal acquisition threshold is defined using Eq (7). We used the Monte-Carlo simulation to calculate the probability of detection for each integration time. The table 1 summarizes the probability of detection.

\begin{tabular}{|c|c|c|c|}
\multicolumn{4}{|c|}{ TABLE I. THE PROBABILITY OF DETECTION } \\
\hline & GPS LI C/A & NH Code & TDPC \\
\hline $1 \mathrm{~ms}$ & 0.0123 & 0.0170 & 0.0110 \\
\hline $2 \mathrm{~ms}$ & 0.0184 & 0.0160 & 0.0180 \\
\hline $4 \mathrm{~ms}$ & 0.0761 & 0.0820 & 0.0800 \\
\hline $5 \mathrm{~ms}$ & 0.1475 & 0.1710 & 0.1700 \\
\hline $10 \mathrm{~ms}$ & 0.6869 & 0.6930 & 0.6950 \\
\hline $20 \mathrm{~ms}$ & 0.9973 & 0.9980 & 0.9950 \\
\hline
\end{tabular}

The mean acquisition time is given as [6]:

$$
E\left[T_{a c q u}\right]=(N-1)\left(T_{d}+T_{f a} P_{f a}\right) \frac{\left(2-P_{d}\right)}{2 P_{d}}+\frac{T_{d}}{P_{d}}
$$

Where $N$ is the maximum number of code phase bin to be searched, $T_{d}$ is the dwell time, and $T_{f a}$ is the dwell time due to the false alarm. $N$ is twice the code length because receiver searches the code phase at half chip intervals. $T_{d}$ uses the GPS L1 C / A code period of $1 \mathrm{~ms} . T_{f a}$ is the product of the false alarm penalty $(k)$ and the dwell time $\left(T_{d}\right)$. The false alarm penalty is set to 5 . The mean acquisition time is shown in Table 2 .

B. Signal Tracking

\begin{tabular}{|c|c|c|c|}
\multicolumn{4}{|c}{ TABLE II. THE MEAN ACQUISITION TIME } \\
\hline & GPS LI C/A & NH Code & TDPC \\
\hline $1 \mathrm{~ms}$ & 166.1452 & 119.9268 & 185.9019 \\
\hline $2 \mathrm{~ms}$ & 221.4475 & 254.9729 & 226.4142 \\
\hline $4 \mathrm{~ms}$ & 103.9697 & 96.1932 & 98.7008 \\
\hline $5 \mathrm{~ms}$ & 64.5645 & 54.9855 & 55.3991 \\
\hline $10 \mathrm{~ms}$ & 19.6587 & 19.3952 & 19.3098 \\
\hline $20 \mathrm{~ms}$ & 20.6836 & 20.6547 & 20.7709 \\
\hline
\end{tabular}


We assumed that the signal power is $-158 \mathrm{dBW}$, which is the GPS nominal power. And signal tracking was done at $1 \mathrm{~ms}$ intervals. Since the primary codes are the same, the effect of the secondary code can be compared with the tracking result. The receiver wipe off and trace the secondary code. As a result, code tracking jitter is similar for all three signals. However, due to the code phase error of secondary code, carrier tracking is affected. The secondary code consists of 0,1 or polyphase sequence. The tracking jitter was about two times larger than that of the $\mathrm{C} / \mathrm{A}$ code alone, due to the varying phase per tracking interval. Fig. 3, 4 and 5 show the code tracking results and Fig. 6, 7 and 8 are carrier tracking results.

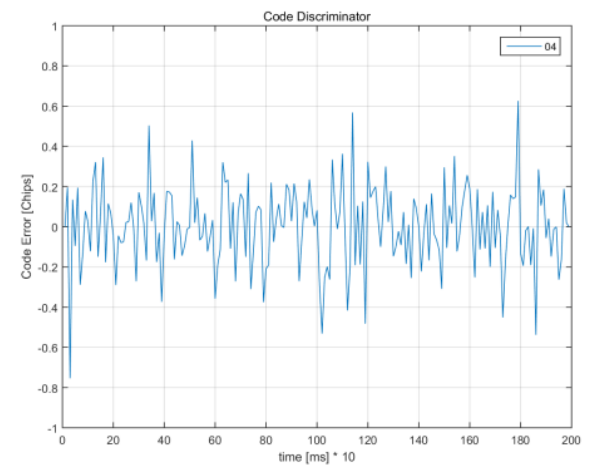

Fig. 3. code tracking jitter(only L1 C/A code).

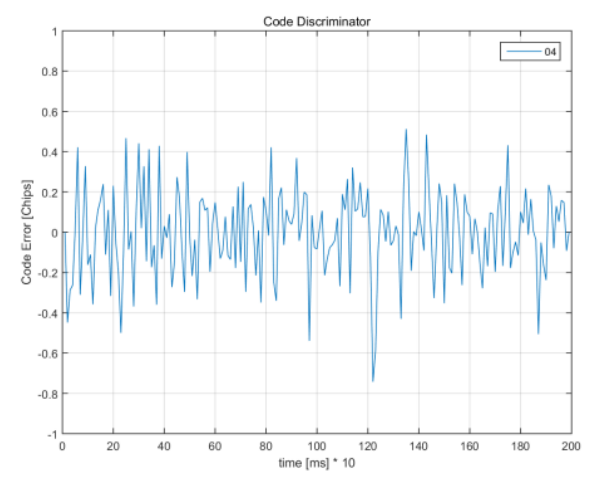

Fig. 4. code tracking jitter(NHsequence).

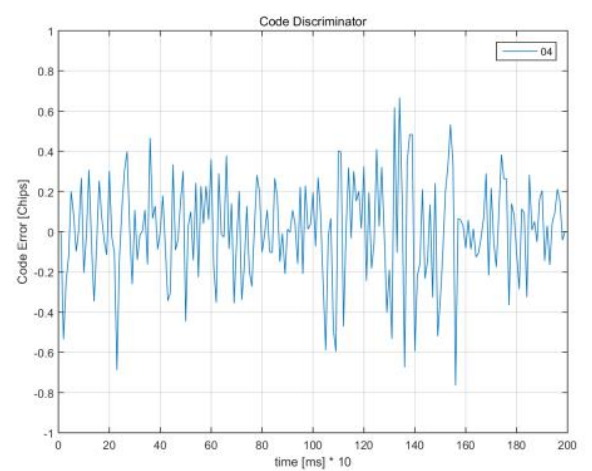

Fig. 5. code tracking jitter(TDPC). 


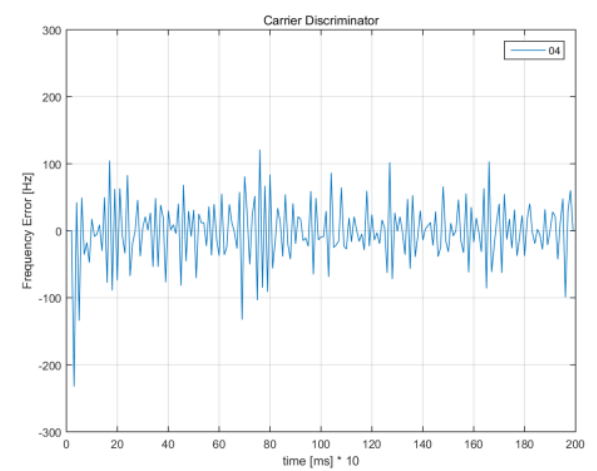

Fig. 6. carrier tracking jitter(only L1 C/A code).

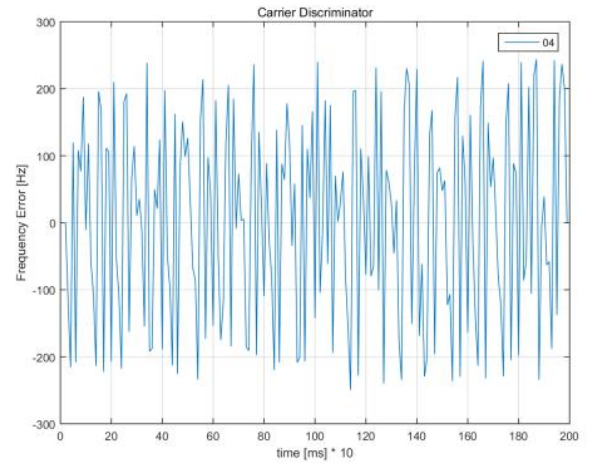

Fig. 7. carrier tracking jitter(NH sequence).

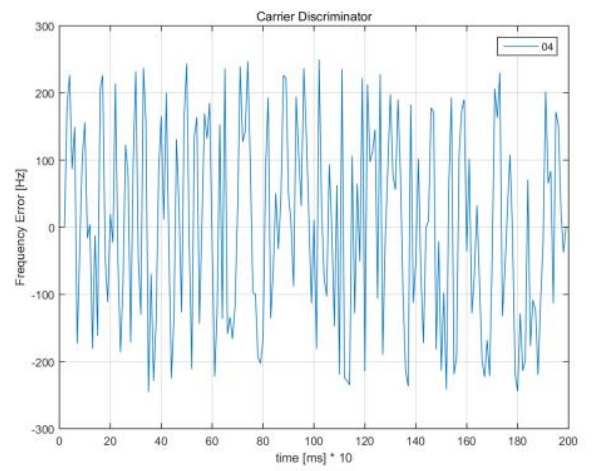

Fig. 8. carrier tracking jitter(TDPC).

\section{CONCLUSION}

In this paper, signal acquisition and tracking simulation were performed by adopting tiered code structure. In order to compare the signal acquisition and signal tracking performance according to the type of the secondary code, GPS L1 C / A code is used as the primary code and the NH code and the TDPC are adopted as the secondary code. The signal acquisition simulation was performed with a signal power $10 \mathrm{~dB}$ lower than the GPS nominal power, and all three cases showed similar results. We used the probability of detection and the mean acquisition time as signal acquisition performance indicators. Although the acquisition performance of the three signal signals was similar, tiered codes using secondary codes were superior in the probability of detection and the mean acquisition time. In the signal tracking simulation, the code tracking performance was similar. However, due to the effect of the secondary code changing at each tracking interval, the carrier tracking jitter has doubled in the tiered code structure. In the future, we will analyze the probability of detection considering the effects of bit transition and Doppler frequency. 


\section{REFERENCES}

[1] H. Li, M. Lu, and Z. Feng, "Partial-correlation-result reconstruction technique for weak global navigation satellite system long pseudo-noise-code acquisition,”, IET Radar Sonar Navig., 2011, vol. 5, no. 7, pp. 731-740.

[2] J. Leclère, C. Botteron, and P. A. Farine, "Acquisition of modern GNSS signals using a modified parallel code-phase search architecture", Signal Process., 2014, vol. 95, pp. 177-191.

[3] S. K. Han, J. Kim, J. K. Kim, A. Han, K. Kim, K. Song, S.-J. Lee, and J. M. Ahn, "Frequency ambiguity free tiered-differential polyphase codes for GNSS signal design," Institution of Engineering and Technology (IET) Electronic Letters, 2017, vol. 53, no. 9, pp. 598-600.

[4] D. C. Chu, "Polyphase Codes with Good Periodic Correlation Properties", IEEE Trans. Info. Theory, 1972, pp. 531-532.

[5] China Satellite Navigation Office, "BeiDou Navigation Satellite System Signal In Space Interface Control Document Open Service Signal B1C (Version 1.0)," 2017.

[6] S. H. Park, I. H. Choi, S. J. Lee, Y. B. K, "A Novel GPS Initial Synchronization Scheme using Decomposed Differential Matched Filter", Proceedings of the 2002 National Technical Meeting of The Institute of Navigation, 2002, pp. 246-253 